\title{
Polynomial Root-MUSIC Algorithm for Efficient Broadband Direction Of Arrival Estimation
}

\author{
William Coventry, Carmine Clemente, and John Soraghan \\ University of Strathclyde, CESIP, EEE, 204, George Street, G1 1XW, Glasgow, UK \\ E-mail: william.coventry, carmine.clemente, j.soraghan- @ strath.ac.uk
}

\begin{abstract}
The popular MUSIC algorithm has been recently extended to broadband scenarios through the use of polynomial matrix methods and polynomial eigenvalue decomposition algorithms, producing impressive results, at the cost of a high computational complexity. This is due to the heuristic angular search stage of the algorithm. Through the extension of the popular narrowband variant of the MUSIC algorithm, RootMUSIC, we aim to significantly reduce the computational cost of spatio-spectral polynomial MUSIC algorithm by eliminating this searching requirement. The performance of both algorithms are analysed and compared, demonstrating a similar performance with a significant reduction in computation time.
\end{abstract}

\section{INTRODUCTION}

The Multiple Signal Classification (MUSIC) algorithm is a popular super-resolution DoA estimation technique for narrowband sources. Due to its popularity, there is a strongly developed framework surrounding the algorithm. This includes many variations, one being the Root-MUSIC algorithm [1]. However, MUSIC and most derivatives are reliant upon exploiting the eigenstructure of a spatial-only covariance matrix. The direct use of this spatial-only covariance matrix is inappropriate in a broadband scenario as time delay across the array can no longer be treated as a phase shift of the carrier, but rather as a linear phase shift across all frequencies [2].

Broadband DoA algorithms based on coherent signal subspace methods coherently combine spatial covariance matrices at separate frequency bins in to a single spatial covariance matrix at a single frequency bin. This new spatial covariance matrix will have the same structure as the narrowband case, thus conventional MUSIC algorithm and variants can be used [3]. While simple, coherent signal subspace methods have drawbacks.

The method of coherently combining spatial covariance matrices at separate frequency bins into a single matrix requires a transformation matrix to 'pre-steer' the data [3]. The generation of this transformation matrix requires knowledge of the sources DoA a priori, thus a preliminary DoA estimate is required before this coherent, high resolution DoA estimation step.

Recent polynomial matrix methods, such as the polynomial eigenvalue decomposition (PEVD), provide an elegant solution in many broadband sensor array signal processing applications, such as beamforming [4] and DoA estimation. The Polynomial MUSIC (PMUSIC) algorithm is a super-resolution DoA estimator for broadband signals [5]. This algorithm is able to achieve very good performance, but with a high computational cost.
In this paper, we propose a generalisation of the narrowband Root-MUSIC algorithm in [1] to broadband scenarios via the use of polynomial matrix methods and the PEVD in effort to significantly reduce computation time.

The remainder of this paper is organised as follows; Section II discusses the data model used for broadband sources illuminating a uniform linear array, and identifies the difficulties in broadband direction finding. Section III introduces the polynomial space-time covariance matrix and the polynomial eigenvalue decomposition. Sections IV and V introduce the SSP-MUSIC, and Root SSP-MUSIC algorithms. Section VI analyses and compares the performance of these algorithms.

Notation. To keep with standard notation, vectors and matrices are denoted by bold-lower and upper case variables respectively, e.g. $\boldsymbol{a}$ and $\boldsymbol{A}$. Polynomial vectors and matrices are denoted as $\boldsymbol{a}(z)$ and $\boldsymbol{A}(z)$. The $z$-transform of a variable $a(n)$ is denoted as $a(n) \circ-\bullet a(z)$. Convolutions are denoted by the $\otimes$ operator.

\section{SignAl MODEL}

Let us consider a multi-antenna array of $M$ omnidirectional elements in the far field of $P$ broadband sources through a non dispersive medium. The received signal at the array is modelled as the superposition of the $P$ steered sources, plus additive noise, which is assumed to be uncorrelated, white, and Gaussian [5].

For a uniform linear array, the wavefront delay between adjacent antenna elements, $\tau_{p}$, is calculated as:

$$
\tau_{p}=\frac{d \sin \left(\theta_{p}\right)}{c}
$$

Where $d$ is the inter element spacing, $\theta_{p}$ is the direction of arrival of the $p^{t h}$ source, and $c$ is the speed of light.

If a source is narrowband, the complex envelope is approximately constant across the array manifold. This reduces the problem to a time-shifted sinusoid, i.e. a phase shift of the carrier [6]. This is an important approximation in the narrowband algorithms as this allows the formation of a simple spatial only covariance matrix at a single frequency bin.

For broadband sources, the inter-element time delay cannot be approximated by a phase shift of the carrier, and must be considered as a linear phase shift across all frequencies. This leads to the following convolutive mixture model [2]: 


$$
\boldsymbol{x}(n)=\sum_{p=1}^{P}\left[\boldsymbol{a}_{\boldsymbol{p}} \otimes s_{p}(n)\right]+\boldsymbol{\nu}(n)
$$

Where $\boldsymbol{x}(n)$ is the sampled vector of antenna signals, $\boldsymbol{a}_{\boldsymbol{p}}$ is the broadband steering vector for the $p^{t h}$ source, $s_{p}(n)$ is the $p^{t h}$ sampled source signal, and $\boldsymbol{\nu}(n)$ is the additive noise vector. This linear phase shift can be modelled as an ideal fractional delay FIR filter, thus the broadband steering vector for the $p^{t h}$ source can be modelled as a vector of these ideal filters, where the delay is an integer multiple of $\tau_{p}$.

$$
\boldsymbol{a}_{\boldsymbol{p}}=\left[\begin{array}{c}
\delta\left[n-0 \tau_{p}\right] \\
\delta\left[n-1 \tau_{p}\right] \\
\vdots \\
\delta\left[n-(M-1) \tau_{p}\right]
\end{array}\right]
$$

Where $\delta[$.$] represents an ideal fractional delay FIR filter.$ This broadband steering vector may also be represented as a polynomial vector via the z-transform. Since the wavefront delay from the $m^{t h}$ antenna is an integer multiple from that of the first, this steering vector can be modelled with a Vandermonde structure.

$$
\boldsymbol{a}_{\boldsymbol{p}}(z)=\left[\begin{array}{c}
\psi_{p}^{0}(z) \\
\psi_{p}^{1}(z) \\
\vdots \\
\psi_{p}^{(M-1)}(z)
\end{array}\right]
$$

Where $\psi_{p}(z)$ is the impulse response of the ideal fractional delay filter with delay $\tau_{p}$. This Vandermonde structure is important when forming the Root SSP-MUSIC algorithm, which will be discussed in Section V. These ideal fractional delay FIR filters, $\delta[n-\tau]$, can be approximated with reasonable accuracy using either appropriately windowed sinc filters, or more complex filter bank techniques [7].

\section{SPACE-TIME COVARIANCE MATRIX AND PEVD}

Due to the convolutive mixture model in equation 2 , a range of temporal correlations need to be considered in addition to spatial correlations. This leads to the definition of the spacetime covariance matrix, $\boldsymbol{R}_{\boldsymbol{x} \boldsymbol{x}}(z)$ [2]

$$
\boldsymbol{R}_{\boldsymbol{x} \boldsymbol{x}}(z)=\sum_{\tau=-\infty}^{\infty} \boldsymbol{R}_{\boldsymbol{x} \boldsymbol{x}}(\tau) z^{-\tau}
$$

Where $\boldsymbol{R}_{\boldsymbol{x} \boldsymbol{x}}(\tau)=E\left[\boldsymbol{x}(n) \boldsymbol{x}^{H}(n-\tau)\right]$. The process is assumed to be stationary and ergodic, thus this expectation operator can be calculated through temporal averaging. This space time covariance matrix may also be expressed as:

$$
\begin{gathered}
\boldsymbol{R}_{\boldsymbol{x} \boldsymbol{x}}(z)=\boldsymbol{A}(z) \boldsymbol{R}_{\boldsymbol{s s}}(z) \tilde{\boldsymbol{A}}(z)+\sigma_{\nu}^{2} \boldsymbol{I} \\
\boldsymbol{R}_{s s}(\tau)=E\left[\boldsymbol{S}(n) \boldsymbol{S}^{H}(n-\tau)\right]
\end{gathered}
$$

Where $\boldsymbol{A}(z)$ is a matrix of polynomial steering vectors, and $\boldsymbol{R}_{\boldsymbol{s s}}(z) \bullet-\circ \boldsymbol{R}_{s s}(\tau)$ is the source cross spectral density matrix.
This matrix will be full rank under the assumption that all sources are uncorrelated [8].

By construction, the space-time covariance matrix possesses the required para-hermitian property $\left(\boldsymbol{R}_{\boldsymbol{x} x}(z)=\right.$ $\tilde{\boldsymbol{R}}_{\boldsymbol{x} \boldsymbol{x}}(z)$ ) for the SBR2 [2] or SMD [9] polynomial eigenvalue decomposition algorithms. When decomposed to its paraunitary eigenvectors $\boldsymbol{U}(z)$, and polynomial eigenvalues $\boldsymbol{\Lambda}(z)$, the space-time covariance matrix will take the form

$$
\boldsymbol{R}_{\boldsymbol{x} \boldsymbol{x}}(z)=\boldsymbol{U}(z) \boldsymbol{\Lambda}(z) \tilde{\boldsymbol{U}}(z)
$$

This can be further partitioned into signal and noise subspaces. Assuming all sources illuminating the array are uncorrelated, the source cross spectral density matrix will be of rank $P$, thus there will be $P$ significant eigenvalues, indicating the dimensions of the signal subspace.

$$
\boldsymbol{R}_{\boldsymbol{x} \boldsymbol{x}}(z)=\left[\begin{array}{ll}
\boldsymbol{U}_{\boldsymbol{s}}(z) & \boldsymbol{U}_{\boldsymbol{n}}(z)
\end{array}\right]\left[\begin{array}{ll}
\boldsymbol{\Lambda}_{\boldsymbol{s}}(z) & \\
& \boldsymbol{\Lambda}_{\boldsymbol{n}}(z)
\end{array}\right]\left[\begin{array}{c}
\tilde{\boldsymbol{U}}_{\boldsymbol{s}}(z) \\
\tilde{\boldsymbol{U}}_{\boldsymbol{n}}(z)
\end{array}\right]
$$

These polynomial eigenvalues are representative of power spectral densities, and can thus be evaluated for $z=e^{j \Omega}$, where $\Omega$ is the normalised frequency.

\section{SSP-MUSIC ALGORITHM}

The rationale behind the polynomial MUSIC algorithm is to exploit the orthogonality between signal and noise subspaces. It is clear that the polynomial steering vectors will form part of the signal subspace, and are thus orthogonal to that of the noise. Similar to narrowband MUSIC, the generalised quantity $\Gamma(\theta, z)$, can be formed [5]:

$$
\Gamma(\theta, z)=\tilde{\boldsymbol{a}}(\theta, z) \boldsymbol{U}_{\boldsymbol{n}}(z) \tilde{\boldsymbol{U}}_{\boldsymbol{n}}(z) \boldsymbol{a}(\theta, z)
$$

with $\boldsymbol{a}(\theta, z)$ being the test steering vector. The goal is to solve the above equation for $\Gamma(\theta, z)=0$. The conventional method involves forming the MUSIC spatio-spectrum, $P_{S S P}(\theta, \Omega)$, by heuristically searching across a range of frequencies and angles. The peaks in this spatio-spectrum will coincide the solutions for $\Gamma(\theta, z)=0$.

$$
P_{S S P}(\theta, \Omega)=\frac{1}{\Gamma(\theta, z)} \mid z=e^{j \Omega}
$$

While this method yields good results, it is moderately expensive in terms of its computational cost. This is due to the many convolutions required for polynomial matrix multiplication in the spatio-spectral search.

\section{Root SSP-MUSIC Algorithm}

The Root Polynomial MUSIC algorithm aims to avoid the many convolution stages required in the above spatiospectral search. By exploiting the Vandermonde structure of the steering vector for a uniform linear array, $\Gamma(\theta, z)$ may also be expressed as a polynomial of $\psi(z)$ 


$$
\begin{aligned}
\Gamma(z) & =\tilde{\boldsymbol{a}}(z) \boldsymbol{U}_{\boldsymbol{n}}(z) \tilde{\boldsymbol{U}}_{\boldsymbol{n}}(z) \boldsymbol{a}(z)=0 \\
& =\tilde{\boldsymbol{a}}(z) \boldsymbol{C}(z) \boldsymbol{a}(z)
\end{aligned}
$$

where $\boldsymbol{C}(z)=\boldsymbol{U}_{\boldsymbol{n}}(z) \tilde{\boldsymbol{U}}_{\boldsymbol{n}}(z)$. Recall that $\psi(z)$ is representative of an ideal all-pass fractional delay FIR filter with delay $\tau$, and its time reversed conjugate, $\psi^{*}\left(z^{-1}\right)$ will have a fractional delay $-\tau$, thus $\psi(z) \psi^{*}\left(z^{-1}\right) \approx 1$. This allows us to express $\Gamma(\psi(z))$ as a Laurent polynomial containing $2 M-1$ coefficients, which are calculated as the sum of the sub-diagonals of $\boldsymbol{C}(z)$, i.e.

$$
\Gamma(\psi(z))=\sum_{l=-(M-1)}^{M-1} b_{l}(z) \psi^{l}(z)
$$

Where $b_{l}$ is the sum of $l^{\text {th }}$ diagonal of $C(z)$.

Note that $\Gamma(\psi(z))$ is a polynomial of $\psi(z)$, while $\psi(z)$ is polynomial of $z$, as such standard polynomial root finding algorithms cannot be directly applied to find the solutions for $\psi(z)$. Recall that $\psi(z)$ is a fractional delay FIR filter, representing the inter-element delay for the source(s) present.

This problem can be solved in the frequency domain by evaluating the polynomials of $z$ for $z=e^{j \Omega}$. Thus equation 13 becomes:

$$
\Gamma\left(\psi\left(e^{j \Omega}\right)\right)=\sum_{l=-(M-1)}^{M-1} b_{l}\left(e^{j \Omega}\right) \psi^{l}\left(e^{j \Omega}\right)
$$

Conventional polynomial rooting algorithms can now be applied to each evaluated frequency. As $\psi(z) \bullet-\circ \delta[n-\tau]$ is representative of an all-pass fractional delay FIR filter, with delay $\tau$ and unity gain, the $P$ roots closest to the unit circle are indicative of the DoA of the $P$ sources. The direction of arrival of the $p^{t h}$ source can be estimated via

$$
\hat{\theta}_{p}\left(e^{j \Omega}\right)=\sin ^{-1}\left[\frac{c \arg \left(q_{p}\left(e^{j \Omega}\right)\right)}{\Omega d}\right]
$$

Where $q_{p}\left(e^{j \Omega}\right)$ is the root relating to the $p^{t h}$ source at frequency $\Omega$.

\section{Performance Analysis}

To analyse and compare the performance of the SSPMUSIC and Root SSP-MUSIC algorithms, parameters such as resolution, accuracy and computational time of the algorithms are evaluated through simulations.

\section{A. Spatio-Spectral Estimation}

In the simulated scenario, two wideband sources are present in anechoic conditions with directions of arrival $30^{\circ}$ and $-40^{\circ}$ in the frequency band $\Omega \in[0.3 \pi, 0.7 \pi]$. The received SNR is $5 \mathrm{~dB}$. The spatial covariance matrix, $\boldsymbol{R}_{\boldsymbol{x} \boldsymbol{x}}(\tau)$ can be estimated via temporal averaging:

$$
\boldsymbol{R}_{\boldsymbol{x} \boldsymbol{x}}(\tau) \approx \frac{1}{N} \sum_{n=1}^{N} x(n) x^{H}(n-\tau)
$$

The polynomial space-time covariance matrix is calculated via the $z$-transform of the spatial covariance matrix

$$
\boldsymbol{R}_{\boldsymbol{x} \boldsymbol{x}}(z)=\sum_{\tau=-W}^{W} \boldsymbol{R}_{\boldsymbol{x} \boldsymbol{x}}(\tau) z^{-\tau}
$$

Note that equation 17 differs from 5 as the summation is now limited to $W$. The value of $W$ should be determined experimentally such that $\boldsymbol{R}_{\boldsymbol{x} \boldsymbol{x}}(\tau) \approx 0$ for $|\tau|>W$ [2]. As mentioned in Section III, this polynomial matrix possesses the required para-Hermitian property, and can thus be decomposed into its polynomial eigenvalues, $\boldsymbol{\Lambda}(z)$, and para-unitary eigenvectors $\boldsymbol{U}(z)$. For these simulations, the MSME-SMD PEVD [10] algorithm is used.

Evaluating the polynomial eigenvalues for $z=e^{j \Omega}$ yields the power spectral density of the eigenvalues. Similarly to the narrowband EVD on a spatial covariance matrix, the number of significant eigenvalues determine the dimensions of the signal subspace, and thus number of uncorrelated sources present. In addition, the eigenvalue power spectral density also contains spectral information on the uncorrelated sources present.

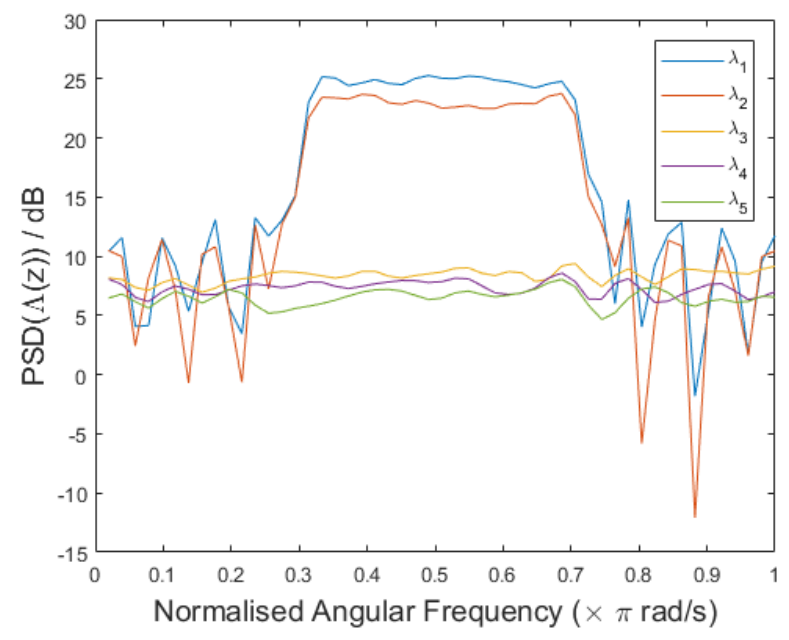

Figure 1: Polynomial Eigenvalue PSD

Figure 1 demonstrates the power spectral density (PSD) of the eigenvalues for this particular scenario. Through a threshold of $20 \mathrm{~dB}$, it is clear that there are two significant eigenvalues present with spectral content in the band $\Omega \in$ $[0.3 \pi, 0.7 \pi]$.

The two significant eigenvalues determine the dimensions of the signal subspace, and thus that of the noise. The SSPMUSIC algorithm from (11) is performed using this determined subspace. Similarly to the model, the elements of the polynomial steering vectors used are the Hann windowed sinc fractional delay FIR filters, with a length of 31 elements. 


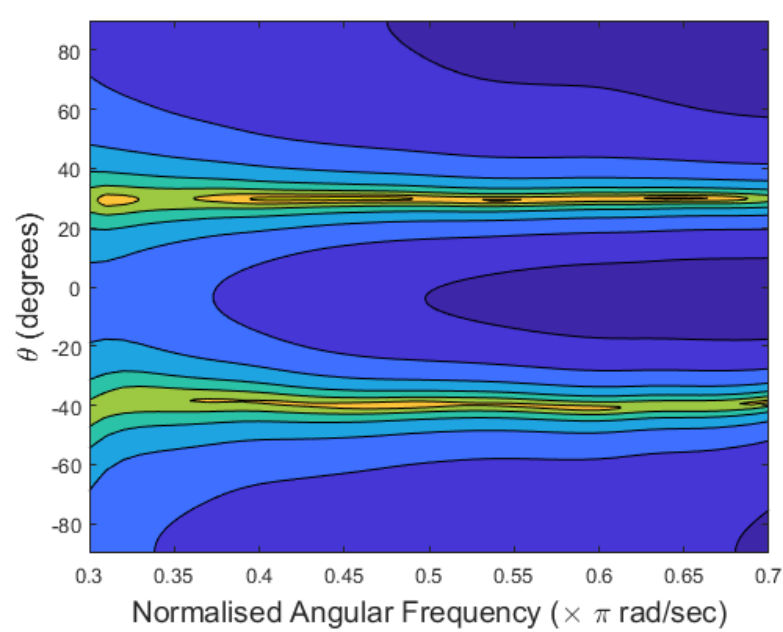

Figure 2: Polynomial MUSIC Spatio-Spectrum

Figure 2 shows the estimated spatio-spectrum with the SSP-MUSIC algorithm and correctly estimates the direction of arrival of the two sources at $-40^{\circ}$, and $30^{\circ}$. As the eigenvalue PSD implies there are no sources outside the band $\Omega \in[0.3 \pi, 0.7 \pi]$, the spatio-spectrum is only estimated for this region.

Applying the Root SSP-MUSIC algorithm on the same data set yields the spatial spectrum in Figure 3

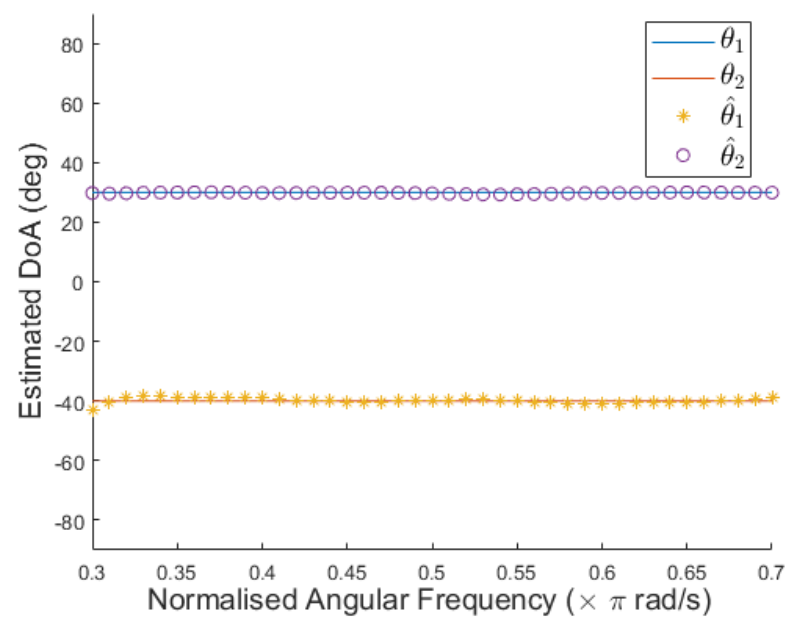

Figure 3: Root-Polynomial MUSIC Spatio-Spectrum

While there is no amplitude information in this spatiospectrum, the estimated directions of arrival coincides with the peaks of the SSP-MUSIC spatio-spectrum, and correctly identifies the direction of arrival of the two sources as $-40^{\circ}$, and $30^{\circ}$.

\section{B. SNR Performance}

A Monte-Carlo simulation was performed to analyse the performance of both algorithms under different SNRs. For each DoA estimate, the DoA of the source was chosen at random from a uniformly distributed set of possible DoAs, $\theta_{p} \in\left[-50^{\circ}, 50^{\circ}\right]$. For each SNR, 100 runs with randomised DoAs were performed. Figure 4 displays the mean squared error of the estimators and it is easy to see that both the SSPMUSIC and Root SSP-MUSIC algorithms perform similarly under different signal to noise ratios, with an MSE $<0.05$ for signal to noise ratios $\geq-4 \mathrm{~dB}$.

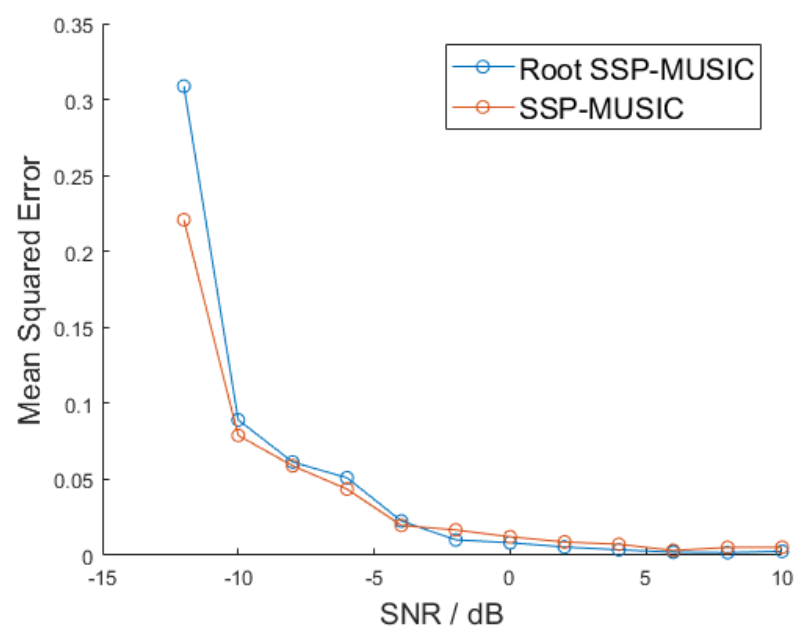

Figure 4: MSE for Monte Carlo simulation at specific SNRs

\section{Computational Cost}

The heuristic angular search of the SSP-MUSIC algorithm is an expensive computational task. This is due to the polynomial matrix multiplications when calculating $\Gamma(\theta, z)$. The Root SSP-MUSIC algorithm replaces this search with finding the roots of a polynomial. Both algorithms, however still require to evaluate polynomials to estimate the spatio-spectrum.

The computer performing this simulation utilised the following hardware and software. Intel core i7-6700 quad-core processor, 16 Gb DDR4 RAM, 256 Gb SSD, Windows 764 bit, MATLAB R2016b.

Table I: Normalised Mean Computation time for SSP-MUSIC and Root SSP-MUSIC algorithm

\begin{tabular}{c|c|c|c|c}
$N_{f}$ & 30 & 60 & 120 & 240 \\
\hline \hline Mean Norm. SSP-MUSIC Comp Time & 1 & 1.04 & 1.124 & 1.268 \\
Mean Norm. Root SSP-MUSIC Comp Time & 0.039 & 0.054 & 0.079 & 0.131
\end{tabular}

The mean normalised simulation time from 100 runs of both algorithms is displayed in Table I. All values are normalised to the SSP-MUSIC computation time for 30 frequency evaluation points. These results show the Root SSP-MUSIC algorithm is considerably faster in computation time, but has a stronger scaling with the number of frequencies evaluated, thus for a large number of frequency points, both algorithms will have similar complexity.

\section{CONCLUSION}

In this paper, an extension of the Root-MUSIC algorithm to broadband scenarios is introduced using polynomial matrix 
methodologies. Forming a more computationally efficient solution for the problem of broadband direction finding in the context of uniform linear arrays. Through simulation results, we have demonstrated that both standalone SSP-MUSIC and Root-SSP MUSIC algorithms perform similarly, yet with a substantial reduction in computational cost with the Root SSPMUSIC algorithm, especially for searches on few evaluated frequency points.

\section{ACKNOWLEDGMENT}

This work was supported by Leonardo MW Ltd

\section{REFERENCES}

[1] R. Kumaresan and D. W. Tufts, "Estimating the angles of arrival of multiple plane waves," IEEE Transactions on Aerospace and Electronic Systems, vol. AES-19, no. 1, pp. 134-139, Jan 1983.

[2] J. G. McWhirter, P. D. Baxter, T. Cooper, S. Redif, and J. Foster "An evd algorithm for para-hermitian polynomial matrices," IEEE Transactions on Signal Processing, vol. 55, no. 5, pp. 2158-2169, May 2007.

[3] H. Wang and M. Kaveh, "Coherent signal-subspace processing for the detection and estimation of angles of arrival of multiple wideband sources," IEEE Transactions on Acoustics, Speech, and Signal Processing, vol. 33, no. 4, pp. 823-831, Aug 1985.

[4] S. Weiss, S. Bendoukha, A. Alzin, F. K. Coutts, I. K. Proudler, and J. Chambers, "Mvdr broadband beamforming using polynomial matrix techniques," in 2015 23rd European Signal Processing Conference (EUSIPCO), Aug 2015, pp. 839-843.

[5] M. A. Alrmah, S. Weiss, and S. Lambotharan, "An extension of the MUSIC algorithm to broadband scenarios using a polynomial eigenvalue decomposition," in 2011 19th European Signal Processing Conference, aug 2011, pp. 629-633.

[6] R. Schmidt, "Multiple emitter location and signal parameter estimation," IEEE Transactions on Antennas and Propagation, vol. 34, no. 3, pp. 276-280, mar 1986.

[7] M. Alrmah, S. Weiss, and J. McWhirter, "Implementation of accurate broadband steering vectors for broadband angle of arrival estimation," in IET Intelligent Signal Processing Conference 2013 (ISP 2013), dec 2013, pp. 1-6.

[8] W. Coventry, C. Clemente, and J. Soraghan, "Enhancing Polynomial MUSIC Algorithm for Coherent Broadband Sources Through Spatial Smoothing," in 25th European Signal Processing Conference (EUSIPCO), 2017, pp. 2517-2521.

[9] S. Redif, S. Weiss, and J. G. McWhirter, "Sequential Matrix Diagonalization Algorithms for Polynomial EVD of Parahermitian Matrices," IEEE Transactions on Signal Processing, vol. 63, no. 1, pp. 81-89, jan 2015.

[10] J. Corr et al., "Multiple shift maximum element sequential matrix diagonalisation for parahermitian matrices," in 2014 IEEE Workshop on Statistical Signal Processing (SSP), June 2014, pp. 312-315. 